\title{
Intimate partner violence victimization and HIV infection among men who have sex with men in Shanghai, China
}

\author{
Ying Liu ${ }^{1,2,3}$, Yuyan Zhang ${ }^{3}$, Zhen Ning $^{4}$, Huang Zheng ${ }^{5}$, Yingying Ding ${ }^{1,2}$, Meiyang Gao ${ }^{1}$, \\ Frank Y. Wong ${ }^{1,6,7}, \mathrm{Na} \mathrm{He}^{1,2, *}$ \\ ${ }^{1}$ Department of Epidemiology, School of Public Health, and the Key Laboratory of Public Health Safety of Ministry of Education, \\ Fudan University, Shanghai, China; \\ ${ }^{2}$ Collaborative Innovation Center of Social Risks Governance in Health, Fudan University, Shanghai, China; \\ ${ }^{3}$ Putuo District Center for Disease Control and Prevention, Shanghai, China; \\ ${ }^{4}$ Shanghai Center for Disease Control and Prevention, Shanghai, China; \\ ${ }_{5}^{5}$ Shanghai Piaoxue Cultural Media Limited, Shanghai, China; \\ ${ }^{6}$ Department of Tropical Medicine, John A. Burns School of Medicine, University of Hawai 'i at Mānoa, USA; \\ ${ }^{7}$ Department of Psychology, College of Social Sciences, University of Hawai ‘i at Mānoa, USA.
}

\begin{abstract}
Summary Intimate partner violence (IPV) and its association with HIV infection among men who have sex men (MSM) in China are not understood. In this study, 732 MSM recruited from Shanghai, China between March and August 2015 were administered with a questionnaire survey and HIV blood testing. IPV victimization was measured by 25 forced-choice items capturing lifetime experience of physical, sexual, psychological, deprivation or neglect, and other forms of violence. Of them, $179(24.3 \%)$ reported having experienced at least one type of IPV victimization. In separate multivariable analyses, sexual violence was associated with age over 35 years $(A O R=\mathbf{0 . 2 6}, 95 \%$ CI: 0.07-1.02), ever had male-to-male commercial sex $(A O R=2.53,95 \% C I: 1.19-5.39)$, and diagnosis of a sexually transmitted infection (STI)

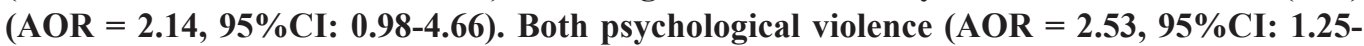
5.12) and deprivation or neglect violence $(A O R=1.75,95 \% \mathrm{CI}: 1.14-2.68)$ were associated with ever had sex with a casual male partner(s). Having experienced at least one type of IPV victimization was significantly associated with ever had sex with a causal partner(s) (AOR $=1.72,95 \%$ CI: $1.15-2.57)$ and ever had a diagnosis of a STI $(A O R=1.80,95 \%$ CI: 1.12 2.88). HIV infection was marginally associated with having experienced any form of IPV victimization. IPV victimization is common among MSM, especially young MSM, in China, although its association with HIV infection is not conclusive in our sample. Nonetheless, our findings highlight the importance of the needs of individualized IPV interventions for certain target risk groups of MSM.
\end{abstract}

Keywords: Intimate partner violence, unprotected sex, HIV infection, MSM, China

\section{Introduction}

HIV has rapidly spread among men who have sex with men (MSM) in China during the past decade. The first national epidemiological survey of HIV among a total of 47,231 MSM from 61 cities from February 2008 to

\footnotetext{
*Address correspondence to:

Dr. Na He, Department of Epidemiology, School of Public Health, and the Key Laboratory of Public Health Safety of Ministry of Education, Fudan University, Shanghai 20032, China

E-mail: nhe@fudan.edu.cn
}

September 2009 revealed an overall prevalence of $4.9 \%$ for HIV infection (1). The percentage of HIV infection in China attributable to homosexual transmission has increased from $12.2 \%$ in 2007 to $27.2 \%$ in 2015 (2). The MSM population has the highest estimate of HIV incidence among all known risk groups (e.g., injection drug users) (3).

There is a large body of epidemiological research documenting the influences of characteristics such as substance misuse and unprotected sex with multiple partners on contracting HIV among MSM in China (4-6). Meanwhile, the international literature reveals 
that intimate partner violence (IPV), which is a subset of interpersonal violence and generally defined as occurring between spouses or other intimate partners, and encompasses multiple domains of violent behavior (e.g., physical, sexual, psychological, financial, stalking) (7), is another major determinant for contracting HIV. However, this body of IPV-HIV literature primarily focuses on women $(8,9)$.

While IPV and its association with HIV infection and/or non-HIV sexually transmitted infections (STIs) among MSM are seldom examined $(10,11)$, the limited literature does suggest that IPV may be a common experience among MSM with a prevalence like or even higher than that among heterosexual women, and have associations with increased odds of substance use, depressive symptoms and engagement in unprotected anal sex $(9,11-15)$.

To the best of our knowledge, there are only two studies examining the relationship between IPV and sexual-related risks among MSM in China. In a study on MSM in Shanghai, China, Dunkle et al. reported a IPV prevalence of $51 \%$ and an association of IPV with more unprotected sex and more sex linked to alcohol and other substance use (16). Another online survey in China reported that $29.8 \%$ of MSM have experienced at least 1 type of IPV, and men who have experienced IPV are more likely to have participated in group sex, to have had sex in exchange for gifts or money, and to selfreport having tested positive for HIV (17). However, the association between IPV victimization and confirmed HIV infection has not been assessed in these two studies, which compromised the reliability of the association between IPV and HIV infection.

To fill this gap, we conducted a cross-sectional study to examine the prevalence of IPV victimization and its association with risk behaviors and HIV infection among MSM in Shanghai, China.

\section{Materials and Methods}

\subsection{Study Design and Participants}

Between March and August 2015, MSM clients accessing services from two voluntary HIV voluntary counseling and testing (VCT) clinics in Shanghai, China were invited to participate in a cross-sectional survey. To be eligible, potential participants must be: (1) born a biological male, (2) ages 18 years or older, (3) have had oral and/or anal sex with another man in the past 12 months, and (4) be able to give written and verbal consent. The study was approved by the Institutional Review Board of Fudan University and written informed consent was obtained from each participant.

\subsection{Data Collection}

Each participant was given a face-to-face interview using a standardized questionnaire to solicit information pertaining to socio-demographic characteristics, sexual behaviors, illicit drug use, and history of STI diagnosis (e.g., syphilis, gonorrhea, chlamydia, genital herpes and genital warts). HIV testing was also performed.

\subsection{Behavioral Measures}

IPV from a male sexual partner (or IPV victimization) was measured by 25 items soliciting lifetime experience of physical, sexual, psychological, deprivation or neglect, and other violence from a male partner. These items were developed in accordance with the definition and typology of violence of the World Health Organization (18), the construction of items used in the WHO Multi-country Study on Women's Health and Domestic Violence against Women (19), with some having previously been validated in the study of IPV and sexual behaviors among MSM in Shanghai and in the nationwide online survey of sexual health among MSM in China $(16,20)$. These 25 forced-choice items cover five domains: physical violence (7 items), sexual violence (3 items), psychological violence (5 items), deprivation or neglect violence ( 7 items), and other violence such as having ever been threatened to reveal their sexuality (3 items).

\subsection{HIV Testing}

Serum samples were screened for anti-HIV IgG antibody by an enzymatic link immunosorbent assay (ELISA) (Wantai Bio-Pharm, Beijing, China). All samples that screened positive for HIV were subject to another antibody testing with another HIV diagnostic kit using ELISA tests. The positive samples were then confirmed by a Western Blot Assay (HIV BLOT 2.2; Genelabs Diagnostics Pte Ltd., Singapore). All laboratory tests were performed according to the manufacturers' instructions.

\subsection{Data Analysis}

The distributions of socio-demographic characteristics, sexual behaviors, illicit drug use, diagnosis of any STIs, and IPV victimization were tabulated as percentages. HIV prevalence was compared using chi-square test or Fisher's exact test when appropriate. Univariable and multivariable logistic regression analyses were performed separately to explore sociodemographic and sexual behavioral correlates of various types of IPV victimization. To examine the correlation between IPV victimization and HIV infection, multiple logistic regression model was employed with adjustment for demographic variables. All statistical analyses were performed using SPSS software (SPSS Inc., Chicago, Illinois, USA). 


\section{Results}

\subsection{Socio-demographic Characteristics}

A total of 760 MSM were approached and identified to be eligible. Of them, $732(96.3 \%)$ participated in the study. Among the participants, $83.9 \%$ were younger than 35 years old; $80.3 \%$ were never married; $59.7 \%$ were migrants (i.e., not born in Shanghai); $78.3 \%$ had college education; and $73.6 \%$ self-identified as gay. Approximately $9.8 \%$ of the participants had ever used illicit drugs (Table 1).

\subsection{Homosexual Behaviors}

Fifteen-percent of the participants had ever engaged in male-to-male commercial sex. Participants reported a high prevalence $(61.9 \%)$ of lifetime experience of sex with a casual male partner(s). Nearly two-thirds $(66.9 \%)$ of the participants reported having had two or more male sex partners in the past year. About 14.1\% reported having ever been diagnosed with a STI.

\subsection{Prevalence of IPV Victimization}

Among the participants, the overall prevalence of any type IPV victimization suffering from a male sexual partner(s) was $24.3 \%$. Specifically, the prevalence was $6.6 \%$ for any form of physical violence; $5.5 \%$ for any form of sexual violence; $8.2 \%$ for any form of psychological violence; $20.1 \%$ for any form of deprivation or neglect violence, and $2.3 \%$ for any form of other violence (Table 2).

\subsection{Correlates of IPV Victimization}

As shown in Table 3, multivariable analyses reveal that MSM (1) who ever had commercial sex with a male sex worker(s) $(\mathrm{AOR}=2.53,95 \% \mathrm{CI}: 1.19$ to $5.39)$ and a diagnosis of any type of STI $($ AOR $=2.14$, 95\% CI: 0.98-4.66) reported more sexual violence; (2) who aged 35 years and older reported less sexual violence ( $\mathrm{AOR}=0.26,95 \% \mathrm{CI}$ : 0.07 to 1.02 ); (3) who ever had sex with a causal male partner(s) in lifetime reported more psychological violence $(\mathrm{OR}=2.53,95 \%$ CI: 1.25 to 5.12 ) and deprivation or neglect violence $(\mathrm{AOR}=1.75,95 \% \mathrm{CI}: 1.14$ to 2.68$)$. Other forms of violence were positively associated with a diagnosis of any STI (AOR $=3.28,95 \%$ CI: 1.05 to 10.27$)$, but negatively associated with having college or advanced education $(\mathrm{AOR}=0.23,95 \% \mathrm{CI}: 0.06$ to 0.93$)$ and MSM who self-identified as gay $(\mathrm{AOR}=0.23,95 \% \mathrm{CI}$ : 0.08 to 0.71 ). Having experience of any type of IPV victimization was positively associated with having sex with a casual male partner(s) (AOR $=1.72,95 \% \mathrm{CI}: 1.15$ to 2.57$)$ and diagnosis of any STI (AOR $=1.80,95 \%$ CI: 1.12 to 2.88 ). Lifetime illicit drug was marginally associated with physical violence.

\subsection{HIV Infection and Its Association with IPV Victimization}

Eighty-five participants (11.6\%) were tested positive for HIV. The HIV prevalence was significantly higher among participants with lower education, HIV-risk taking behaviors including commercial sex, casual sex, multiple partnership and illicit drug use, as well as diagnosis of a STI (Table 1). As shown in Table 2, the HIV prevalence was $11.4 \%$ for those without any IPV, $20.0 \%$ for those with one type of IPV and $9.4 \%$ for those with two or more types of IPV. No separate and significant association was identified between HIV infection and any of the five violence domains using bivariate chi-square test (Table 2). However, there was a marginally significant association between HIV infection and IPV after adjusted for age, education, marital status and sexual identity according to a multivariable logistic regression analysis (Table 4).

\section{Discussion}

In this study we examined IPV among MSM in China, and its association to HIV infection. Our finding reveals a relatively high prevalence of IPV victimization among MSM attending VCT clinics in Shanghai, China. It is similar to the rate reported by the nationwide online survey among MSM in China (17), but lower than the rate among MSM in Shanghai reported by Dunkle et al (10). The possible explanation for the discrepant results between our study and the Dunkle's is that about a half of participants in Dunkle's study were "money boys" (i.e., man who sells sex to man), who may be more vulnerable to IPV than general MSM.

The difference in estimating IPV prevalence may also reflect differences in sampling methodology used in the three studies. We used a clinic-based methodology to recruit MSM, whereas Dunkle et al. used Respondent-driven sampling methodology which has been shown for attracting higher risk populations such as "money boys." Indeed, Ibragimov et al. argue that type of recruitment method used is likely to reach a certain sub-type of MSM with a specific risk profile (21); therefore, these researchers recommend multiple methods are needed to obtain a representative sample of MSM.

Consistent with the known literature(11,22-26), we also found that MSM engaging in high-risk sexual behaviors such as commercial sex and casual sex, or had a history of STIs are more likely to report IPV victimization. Notably, our findings reveal that young MSM reported more sexual violence than older MSM. One possible explanation for this phenomenon might be due to sexual mixing with older male partners among young MSM in Shanghai, partly for emotional, 


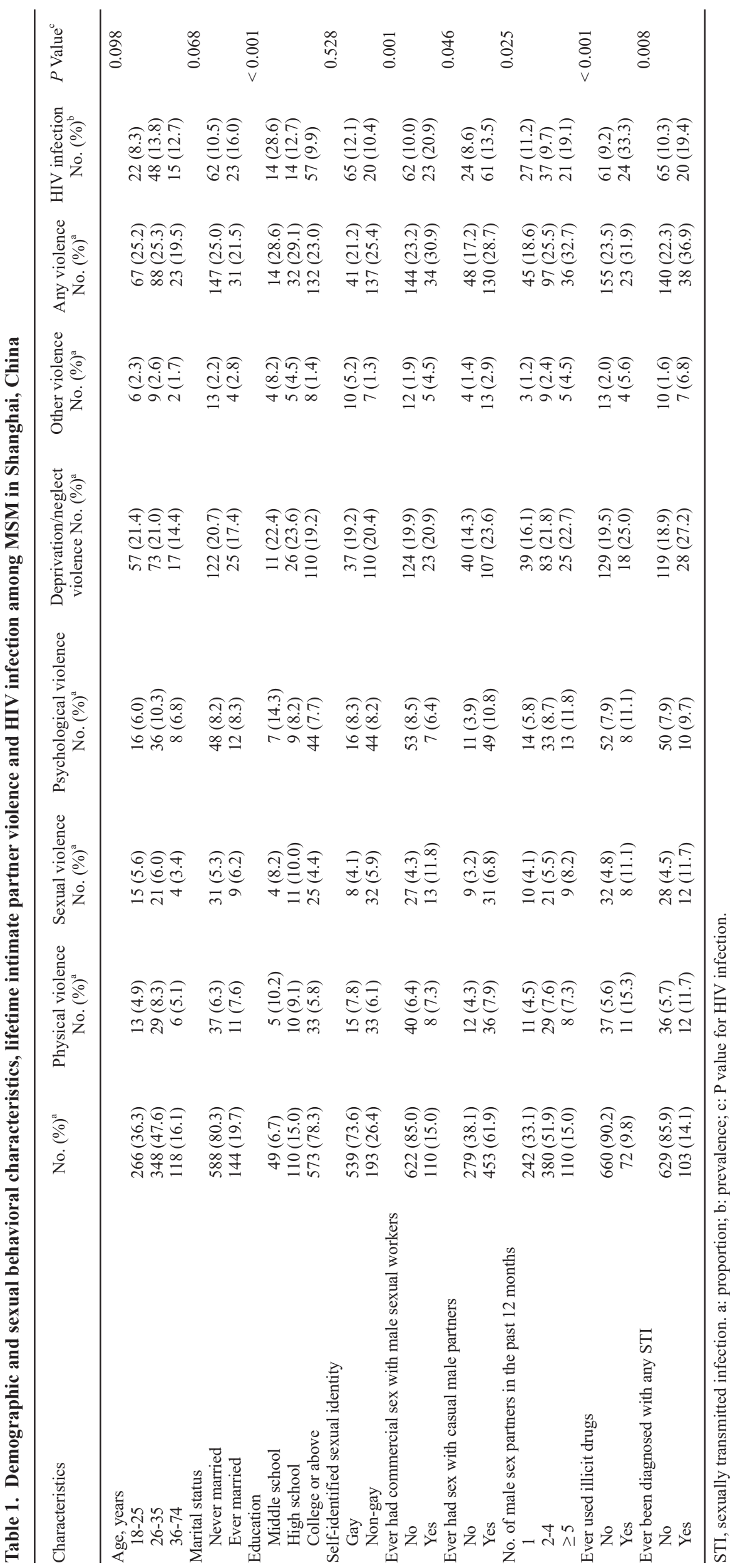


Table 2. Lifetime experience of various type of IPV victimization from male sexual partners among MSM in Shanghai

\begin{tabular}{|c|c|c|}
\hline Type of IPV victimization & No. $(\%)^{\mathrm{a}}$ & HIV infection N No. $(\%)^{\mathrm{b}}$ \\
\hline \multicolumn{3}{|l|}{ Domain 1: Physical violence } \\
\hline Ever been slapped & $24(3.3)$ & \\
\hline Ever been thrown with something & $26(3.6)$ & \\
\hline Ever been pushed or shoved & $25(3.4)$ & \\
\hline Ever been hit with fist or something else could hurt & $18(2.5)$ & \\
\hline Ever been kicked or dragged & $12(1.6)$ & \\
\hline Ever been choked or burnt on purpose & $5(0.7)$ & \\
\hline Ever been threatened to use or actually used a gun, knife or other weapon against you & $3(0.4)$ & \\
\hline Having any physical violence & & $P=0.789$ \\
\hline No & $684(93.4)$ & $80(11.7)$ \\
\hline Yes & $48(6.6)$ & $5(10.4)$ \\
\hline \multicolumn{3}{|l|}{ Domain 2: Sexual violence } \\
\hline Ever been physically forced to have sexual intercourse when you did not want to & $35(4.8)$ & \\
\hline Ever had sexual intercourse when you did not want to because of what he might do & $8(1.1)$ & \\
\hline Ever been forced to do something sexual that degrading or humiliating & $8(1.1)$ & \\
\hline Having any sexual violence & & $P=0.211^{\mathrm{c}}$ \\
\hline No & $692(94.5)$ & $78(11.3)$ \\
\hline Yes & $40(5.5)$ & $7(17.5)$ \\
\hline \multicolumn{3}{|l|}{ Domain 3: Psychological violence } \\
\hline Ever been insulted or made feel bad & $38(5.2)$ & \\
\hline Ever been belittled or humiliated in front of other people & $23(3.1)$ & \\
\hline Ever been scared or intimidated on purpose & $27(3.7)$ & \\
\hline Ever been verbally threatened to harm you physically or emotionally & $17(2.3)$ & \\
\hline Ever been verbally threatened to physically harm someone you care for & $12(1.6)$ & \\
\hline Having any psychological violence & & $P=0.212$ \\
\hline No & $672(91.8)$ & $81(12.1)$ \\
\hline Yes & $60(8.2)$ & $4(6.7)$ \\
\hline \multicolumn{3}{|l|}{ Domain 4: Deprivation or neglect violence } \\
\hline Ever been kept from seeing friends & $31(4.2)$ & \\
\hline Ever been restricted to contact with your family & $9(1.2)$ & \\
\hline Ever insisted on knowing where you are at all times & $82(11.2)$ & \\
\hline Ever been ignored and treated indifferently & $62(8.5)$ & \\
\hline Ever been angry with you if you spoke with another man & $75(10.2)$ & \\
\hline Been often suspicious of your unfaithful & $73(10.0)$ & \\
\hline Ever been expected to ask permission before seeking health care & $25(3.4)$ & \\
\hline Having any deprivation or neglect violence & & $P=0.241$ \\
\hline No & $585(79.9)$ & $72(12.3)$ \\
\hline Yes & $147(20.1)$ & $13(8.8)$ \\
\hline \multicolumn{3}{|l|}{ Domain 5: Other violence } \\
\hline Ever been threatened to reveal your sexuality & $2(0.3)$ & \\
\hline Your property has ever been damaged or destroyed & $12(1.6)$ & \\
\hline Ever been threatened to stop helping you with money or with housing & $9(1.2)$ & \\
\hline Having any other violence & & $P=0.709^{\mathrm{c}}$ \\
\hline No & $715(97.7)$ & $84(11.7)$ \\
\hline Yes & $17(2.3)$ & $1(5.9)$ \\
\hline \multicolumn{3}{|l|}{ No. of above-mentioned types of IPV } \\
\hline 0 & $554(75.7)$ & $63(11.4)$ \\
\hline 1 & $50(6.8)$ & $10(20.0)$ \\
\hline$\geq 2$ & $128(17.5)$ & $12(9.4)$ \\
\hline
\end{tabular}

${ }^{\mathrm{a}}$ : proportion; ${ }^{\mathrm{b}}$ : prevalence; ${ }^{\mathrm{c}}$ : Fisher's exact test.

financial and instrumental support (27). In such relationship, young MSM may be at a disadvantage position and thereby are more likely to be victims of sexual violence. Sex with older male partners may also increase their risk of HIV infection (27).

The relationship between IPV and HIV infection among MSM population has been reported in some but not all studies $(10,28-30)$. In our sample, HIV infection is marginally associated with having experienced any form of IPV victimization. Nonetheless, our descriptive data indicate that higher prevalence of HIV infection is only observed among those with sexual violence compared with those without, suggesting that the link between IPV and HIV infection is mostly related to sexual violence. The discrepancies between studies might be due to the confluence of various types of IPV and its impact on HIV risks. For example, an initial act of physical violence might be likely to escalate sexual violence among intimate partners. Further research is needed to elucidate the dynamics of IPV "penetration" on victimization.

The current study had several limitations. First, all participants were recruited from VCT clinics in Shanghai, limiting the ability to generalize the findings to other MSM populations in China. Second, due to the cross-sectional nature, the temporal order between IPV victimization and HIV infection could not be determined. Third, our data were self-reported, some sensitive topics such as sexual behaviors and IPV victimization experiences might be underreported by participants due to the social desirability; and these were lifetime not recent experiences and behaviors, thereby recall bias could not be neglected particularly for older MSM 


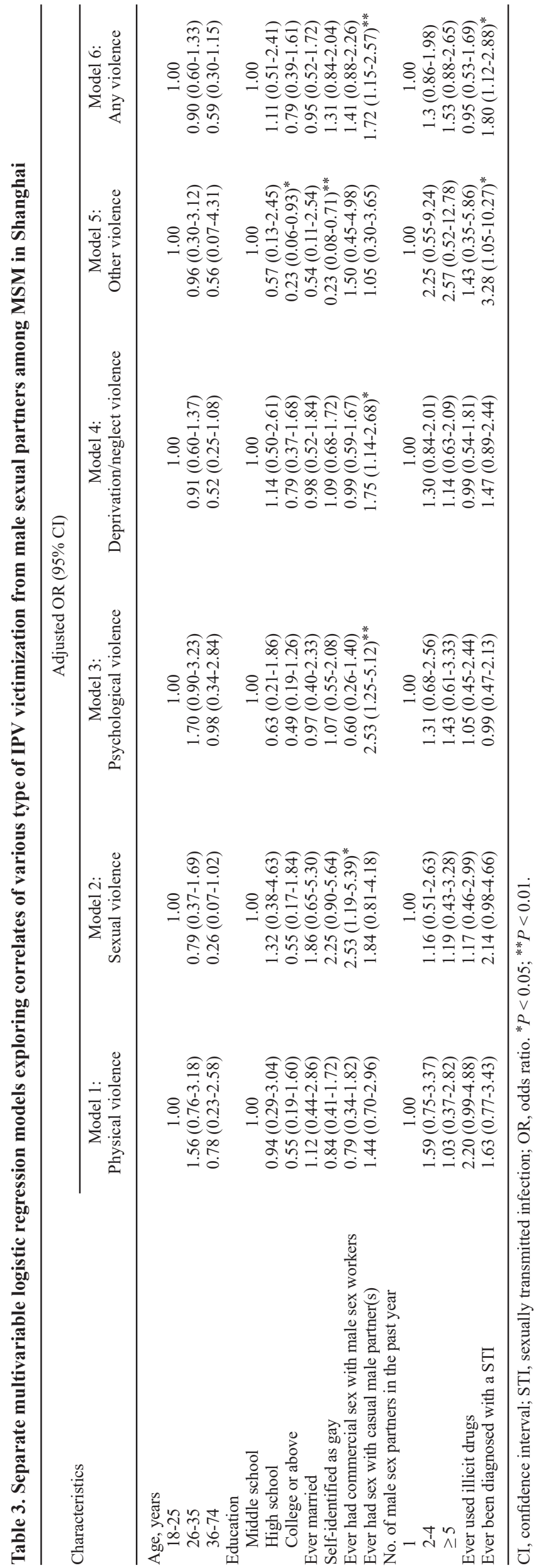

Table 4. Multivariable logistic regression analysis of correlates with HIV infection among MSM in Shanghai

\begin{tabular}{lcr}
\hline Items & Adjusted OR (95\%CI) & $P$ Value \\
\hline Age, years & & \\
$18-25$ & 1.0 & \\
$26-35$ & $1.81(1.04-3.16)$ & 0.037 \\
$\quad 36-74$ & $1.02(0.43-2.41)$ & 0.963 \\
Education & & \\
$\quad$ Middle school & 1.0 & \\
High school & $0.39(0.17-0.92)$ & 0.030 \\
$\quad$ College or above & $0.25(0.12-0.53)$ & $<0.001$ \\
Ever married & $1.58(0.77-3.24)$ & 0.214 \\
Self-identified as gay & $1.66(0.90-3.06)$ & 0.105 \\
No. of types of IPV victimization & & \\
$\quad$ None & 1.0 & \\
1 & $2.01(0.94-4.32)$ & 0.072 \\
$\geq 2$ & $0.74(0.38-1.43)$ & 0.363 \\
\hline
\end{tabular}

$\mathrm{CI}$, confidence interval; OR, odds ratio.

participants.

In conclusion, our findings demonstrate a high prevalence of IPV victimization among MSM particularly young MSM population in China. Our data also show that various types of IPV victimization are linked to different socio-demographic and sexual behavioral characteristics, suggesting different mechanisms of various types of IPV. These, taken together, underscore the need of individualized IPV interventions for certain target risk groups of MSM. Further research is needed to clarify the complex link between IPV, drug use, types of sexual relationship, and HIV/STI infection.

\section{Acknowledgements}

This study was supported by the Shanghai Municipal Health and Family Planning Commission (grant no. GWTD2015S05, 15GWZK0101, 201540066) and the Natural Science Foundation of China (grant no. 81361120385).

\section{References}

1. Wu Z, Xu J, Liu E, et al. HIV and syphilis prevalence among men who have sex with men: A cross-sectional survey of 61 cities in China. Clin Infect Dis. 2013; 57:298-309.

2. National Health and Family Planning Commission of The People's Republic of China. China AIDS Response Progress Report. Beijing, China, 2015.

3. Cui Y, Guo W, Li D, Wang L, Shi CX, Brookmeyer R, Detels R, Ge L, Ding Z, Wu Z. Estimating HIV incidence among key affected populations in China from serial cross-sectional surveys in 2010-2014. J Int AIDS Soc 2016; 19:20609.

4 Nehl EJ, He N, Lin L, Zheng T, Harnisch JA, Ding Y, Wong FY. Drug use and sexual behaviors among MSM in China. Subst Use Misuse. 2015; 50:123-136.

5 Yu F, Nehl EJ, Zheng T, He N, Berg CJ, Lemieux AF, Lin L, Tran A, Sullivan PS, Wong FY. A syndemic including cigarette smoking and sexual risk behaviors among a sample of MSM in Shanghai, China. Drug Alcohol 
Depend. 2013; 132:265-270.

6. Huang ZJ, He N, Nehl EJ, Zheng T, Smith BD, Zhang J, McNabb S, Wong FY. Social network and other correlates of HIV testing: Findings from male sex workers and other MSM in Shanghai, China. AIDS Behav. 2012; 16:858871.

7. Finneran C, Stephenson R. Intimate Partner Violence Among Men Who Have Sex With Men: A Systematic Review. Trauma Violence Abuse. 2013; 14:168-185.

8. Jewkes RK, Dunkle K, Nduna M, Shai N. Intimate partner violence, relationship power inequity, and incidence of HIV infection in young women in South Africa: A cohort study. Lancet. 2010; 376:41-48.

9. Li Y, Marshall CM, Rees HC, Nunez A, Ezeanolue EE, Ehiri JE. Intimate partner violence and HIV infection among women: A systematic review and meta-analysis. J Int AIDS Soc. 2014; 17:18845.

10. Dunkle KL, Jewkes RK, Murdock DW, Sikweyiya Y, Morrell R. Prevalence of Consensual Male-Male Sex and Sexual Violence, and Associations with HIV in South Africa: A Population-Based Cross-Sectional Study. PLoS Med. 2013; 10:e1001472.

11. Buller AM, Devries KM, Howard LM, Bacchus LJ. Associations between intimate partner violence and health among men who have sex with men: A systematic review and meta-analysis. PLoS Med. 2014; 11:e1001609.

12. Duncan DT, Goedel WC, Stults CB, Brady WJ, Brooks FA, Blakely JS, Hagen D. A study of intimate partner violence, substance abuse, and sexual risk behaviors among gay, bisexual, and other men who have sex with men in a sample of geosocial-networking smartphone application users. Am J Mens Health. 2018; 12:292-301.

13. Williams JK, Wilton L, Magnus M, Wang L, Wang J, Dyer TP, Koblin BA, Hucks-Ortiz C, Fields SD, Shoptaw S, Stephenson R, O Cleirigh C, Cummings V. Relation of childhood sexual Abuse, intimate partner violence, and depression to risk factors for HIV among black men who have sex With men in 6 US Cities. Amer J Public Health. $2015 ; 105: 2473-2481$.

14. Rhodes SD, McCoy TP, Wilkin AM, Wolfson M. Behavioral risk disparities in a random sample of selfidentifying gay and non-gay male university Students. J Homosexuality. 2009; 56:1083-1100.

15. Stockman JK, Lucea MB, Campbell JC. Forced sexual initiation, sexual intimate partner violence and HIV risk in women: A global review of the literature. AIDS Behav. 2013; 17:832-847.

16. Dunkle KL, Wong FY, Nehl EJ, Lin L, He N, Huang J, Zheng T. Male-on-male intimate partner violence and sexual risk behaviors among money boys and other men who Have sex with men in Shanghai, China. Sex Transm Dis. 2013; 40:362-365.

17. Davis A, Best J, Wei C, Luo J, Van Der Pol B, Meyerson B, Dodge B, Aalsma M, Tucker J. Intimate partner violence and correlates with risk behaviors and HIV/STI diagnoses among men who have sex with men and men who have sex with men and women in China. Sex Transm Dis. 2015; 42:387-392.

18. World Health Organization (Internet). Definition and typology of violence. http://www. who.int/ violenceprevention/approach/definition/en/ (accessed Dec 20, 2016).

19. Garcia-Moreno C, Jansen HA, Ellsberg M, Heise L, Watts $\mathrm{CH}$. Prevalence of intimate partner violence: Findings from the WHO multi-country study on women's health and domestic violence. Lancet. 2006; 368:1260-1269.

20. Zhang Y, Best J, Tang W, Tso LS, Liu F, Huang S, Zheng H, Yang B, Wei C, Tucker JD. Transgender sexual health in China: A cross-sectional online survey in China. Sex Transm Infect. 2016; pii:sextrans-2015-052350.

21. Ibragimov U, Harnisch JA, Nehl EJ, He N, Zheng T, Ding Y, Wong FY. Estimating self-reported sex practices, drug use, depression, and intimate partner violence among MSM in China: A comparison of three recruitment methods. AIDS Care. 2017; 29:125-131.

22. Kramer SC, Schmidt AJ, Berg RC, Furegato M, Hospers H, Folch C, Marcus U. Factors associated with unprotected anal sex with multiple non-steady partners in the past 12 months: Results from the European MenWho-Have-Sex-With-Men Internet Survey (EMIS 2010). BMC Public Health. 2015; 16:47.

23. Koblin BA, Torian L, Xu G, Guilin V, Makki H, Mackellar D, Valleroy L. Violence and HIV-related risk among young men who have sex with men. AIDS Care. 2006; 18:961-967.

24. Houston E, McKirnan DJ. Intimate partner abuse among gay and bisexual men: Risk correlates and health outcomes. J Urban Health. 2007; 84:681-690.

25. Decker MR, Seage GR, Hemenway D, Gupta J, Raj A, Silverman JG. Intimate partner violence perpetration, standard and gendered STI/HIV risk behaviour, and STI/ HIV diagnosis among a clinic-based sample of men. Sex Transm Infect. 2009; 85:555-560.

26. Raj A, Kidd JD, Cheng DM, Coleman S, Bridden C, Blokhina EA, Krupitsky E, Samet JH. Associations between partner violence perpetration and history of STI among HIV-infected substance using men in Russia. AIDS Care. 2013; 25:646-651.

27. Ding Y, Zhou Y, Liu C, Liu X, He N. Sex with older partners, condomless anal sex and unrecognized HIV infection among Chinese men who have sex with men. AIDS Care. 2018; 30:305-311.

28. Stults CB, Javdani S, Greenbaum CA, Kapadia F, Halkitis PN. Intimate partner violence and substance use risk among young men who have sex with men: The P18 cohort study. Drug Alcohol Depend. 2015; 154:54-62.

29. Wirtz AL, Jumbe V, Trapence G, Kamba D, Umar E, Ketende S, Berry M, Strömdahl S, Beyrer C, Baral SD. HIV among men who have sex with men in Malawi: Elucidating HIV prevalence and correlates of infection to inform HIV prevention. J Int AIDS Soc. 2013; 16(Suppl 3): 18742 .

30. Relf MV, Huang B, Campbell J, Catania J. Gay Identity, Interpersonal violence, and HIV risk behaviors: An empirical test of theoretical relationships among a probability-based sample of urban men who Have Sex with Men. J Assoc Nurses AIDS Care. 2004; 15:14-26.

(Received March 10, 2018; Revised April 22, 2018; Accepted April 23, 2018) 